\title{
Technetium Chemistry in HLW: Role of Organic Complexants
}

(Project Number: 81921)

\section{Principal Investigator}

Nancy J. Hess

Pacific Northwest National Laboratory

P.O. Box 999, MSIN P7-50

Richland, WA 99352

509-376-9808 (phone)

509-372-1632 (fax)

nancy.hess@pnl.gov

\section{Co-Principal Investigators}

David L. Blanchard, Jr.

Pacific Northwest National Laboratory

P.O. Box 999, MSIN P7-25

Richland, WA 99352

509-372-2248 (phone)

509-372-3861 (fax)

dl.blanchard@pnl.gov

James A. Campbell

Pacific Northwest National Laboratory

P.O. Box 999, MSIN P8-08

Richland, WA 99352

509-376-0899 (phone)

509-376-2329 (fax)

james.campbell@pnl.gov

Herman M. Cho

Pacific Northwest National Laboratory

P.O. Box 999, MSIN K8-98

Richland, WA 99352

509-376-2685 (phone)

509-376-2303 (fax)

hm.cho@pnl.gov
Dhanpat Rai

Pacific Northwest National Laboratory P.O. Box 999, MSIN P7-50

Richland, WA 99352

509-373-5988 (phone)

509-372-1632 (fax)

dhan.rai@pnl.gov

Yuanxian Xia

Pacific Northwest National Laboratory

P.O. Box 999, MSIN P7-50

Richland, WA 99352

509-373-5767 (phone)

509-372-1632 (fax)

yuanxian.xia@pnl.gov

Steven D. Conradson

Los Alamos National Laboratory

MST-8, MS G755

Los Alamos, NM 87545

505-667-9584 (phone)

505-667-8021 (fax)

conradson@lanl.gov 


\section{Research Objective}

Technetium complexation with organic compounds in tank waste plays a significant role in the redox chemistry of Tc and the partitioning of Tc between the supernatant and sludge components in waste tanks. These processes need to be understood so that strategies to effectively remove Tc from high-level nuclear waste prior to waste immobilization can be developed and so that longterm consequences of Tc remaining in residual waste after sludge removal can be evaluated. Only limited data on the stability of Tc-organic complexes exists, and even less thermodynamic data on which to develop predictive models of Tc chemical behavior is available. To meet these challenges, we present a research program to study Tc-speciation in actual tank waste using state-of-the-art analytical organic chemistry, separations, and speciation techniques. On the basis of such studies, we will acquire thermodynamic data for the identified Tc-organic complexes over a wide range of chemical conditions in order to develop credible models to predict Tc speciation in tank waste and Tc behavior during waste pretreatment processing and in waste tank residuals.

\section{Research Progress and Implications}

This report summarizes work after 9 months of a 3-year study. Hanford waste tanks AN-102 and T-111 have been selected for initial analysis based on the availability of samples and previous analytical results that suggest high concentrations of Tc and organics. Samples from AN-102 will be analyzed using electrospray-mass spectroscopy to identify the Tc-organic complexes. The oxidation state of Tc in samples from T-111 will be analyzed using x-ray absorption spectroscopy (XAS) at the Stanford Synchrotron Radiation Laboratory.

Technetium in T-111 sludge is resistant to removal using leaching and washing techniques that have been successful in removing pertechnetate from high-level waste sludge in other tanks, which suggests that $\mathrm{Tc}$ is present in a reduced oxidation state and may be complexed with organics. A previous analysis of the organic fraction of T-111 sludge indicated that a large portion of the organic compounds is unique to this tank. Analysis of the Tc K-edge with XAS will allow determination of the oxidation state of Tc in supernantant and sludge fraction of the waste. If sufficiently high concentrations of Tc are encountered, then the complexation of Tc may be possible as well.

Procedures have been developed for running radioactive tank waste samples on the capillary electrophoresis (electrospray) mass spectrometer (CE/MS). These procedures include contacting $\mathrm{AN}-102$ supernatant with ion exchange resins to remove ${ }^{137} \mathrm{Cs}$ and ${ }^{90} \mathrm{Sr}$ to reduce the radiation dose without affecting the complexation of Tc. An enclosure to contain possible radioactive contamination during analysis also has been designed. We currently are investigating analog Tc-organic compounds to study the fragmentation of these complexes in negative ion mode and 
to test the performance of the system. We then will run model Tc-organic compounds prior to analyzing actual tank waste. The application of a CE/MS system to Hanford tank waste will require development of additional methods. Actual tank waste or simulated tank waste is extremely high in salt content. The high salt content tends to suppress the signal during electrospray ionization. Therefore, methods will be evaluated to remove the salts without removing any of the metal-organic complexes prior to analysis using electrospray mass spectrometry.

\section{Planned Activities}

Sludge and supernatant samples from T-111 will be analyzed using XAS at the Stanford Synchrotron Radiation Laboratory in June 2002 to determine the Tc oxidation state. 